\title{
Identification and antimicrobial susceptibility testing of anaerobic bacteria isolated from clinical samples
}

\begin{abstract}
Background/aims: Anaerobic bacteria have attracted the attention of scientists as the causative agents of serious infections since the 19th century. Antibiotic resistance has become a major problem among these bacteria, but antibiotic susceptibility testing has not been standardized yet.

Materials and methods: In this study, anaerobic bacteria isolated from a total of 800 clinical materials sent to our laboratory were evaluated. A total of 69 anaerobic bacteria isolated were identified with VITEK 2 (Biomerieux). Antibiotic susceptibility testing was performed on 23 of 69 isolates with gradient test method.

Results: Identified anaerobic bacteria were Prevotella spp. (19 isolates, 27.5\%), Veillonella spp. (14 isolates, 20.2\%), Bacteroides spp. (9 isolates, 13\%), Peptoniphilus spp. (6 isolates, 8.6\%), Parvimonas spp. (5 isolates, 7.2\%), Fusobacterium spp. (3 isolates, 4.3\%), Actinomyces spp. (3 isolates, 4.3\%), Parabacteroides spp. (2 isolates, 2.8\%), Finegoldia magna (2 isolates, $2.8 \%$ ), Clostridium spp. (2 isolates, $2.8 \%$ ), Propionibacterium spp. (1 isolate, 1.4\%), Peptostreptococcus spp. (1 isolate, 1.4\%), Bifidobacterium spp. (1 isolate, $1.4 \%$ ) and Lactobacillus spp. (1 isolate, 1.4\%). Resistance rates of anaerobic bacteria to metronidazole, imipenem, penicillin, clindamycin and cefoxitin were found as $\% 95.7$, $\% 100, \% 69.6, \% 82.6, \% 95.7$ respectively.
\end{abstract}

Conclusion: Treatment of infections caused by anaerobic microorganisms is usually empirical. Periodic determination of regional susceptibility patterns will help clinicians choose appropriate antibiotics for such infections.

Keywords: anaerobic bacteria, antibiotics resistance, gradient test method, clindamycin, bacterial infections
Volume 8 Issue I - 2020

\author{
Fatma BACALAN,' Fatih ÇAKIR, ${ }^{2}$ Nida \\ ÖZCAN, ${ }^{2}$ Nezahat AKPOLAT ${ }^{2}$ \\ 'Laboratory of Microbiology, Diyarbakır Pediatrics Hospital, \\ Diyarbakır Turkey \\ ${ }^{2}$ Department of Medical Microbiology, Dicle University, \\ Diyarbakır Turkey
}

Correspondence: Nida ÖZCAN, Department of Medical Microbiology, Faculty of Medicine, Dicle University, Diyarbakır, Turkey,Tel +9050527l0103, Email nida.ozcan@dicle.edu.tr

Received: June 02, 2020 | Published: June 15, 2020

\section{Introduction}

Anaerobic bacteria are microorganisms that can live in an oxygenfree environment, but oxygen sensitivity degrees of this heterogeneous group may vary. Obligate anaerobic bacteria require the removal of free oxygen from the environment in order to grow. ${ }^{1}$ Anaerobic bacteria, which are members of the human microbiota, constitute important part of the pathogenic bacteria. Although anaerobic bacteria are the dominant bacteria of the human organism, they cause disease under certain conditions. Anaerobic bacterial infections other than tetanus, gas gangrene, and botulismus are endogenous and often caused by multiple aerobe-anaerobic microorganisms. ${ }^{2,3}$ Transport and cultivation of samples in anaerobic infections require special conditions. Providing anaerobic atmospheric conditions during transport and cultivation are crucial components of anaerobic bacteria isolation. Identifying anaerobic bacteria using conventional methods after isolation is also labor intensive and time consuming. Although difficult and time-consuming, identifying anaerobic bacteria and determining antibiotic susceptibilities are crucial in terms of guiding proper treatment of anaerobic infections. ${ }^{1-3}$ The aim of this study was to isolate anaerobic bacteria from clinical samples and to determine their susceptibility.

\section{Materials and methods}

Approval for this study was obtained from the Non-Interventional Clinical Research Ethics Committee of Dicle University. A total of 800 samples (pleural fluid, abscess material, peritoneal fluid, wound, infected tissue, joint fluid, etc.) sent from Dicle University Hospital clinics to our laboratory with suspicion of anaerobic bacterial infection were examined. While $620(77.5 \%)$ of the samples were transported in the syringe, $180(22.5 \%)$ of the samples were sent to the laboratory via Amies transport medium. Eosin Methylene Blue (EMB) agar and 5\% sheep blood agar (SBA) were used for aerobe cultivation of samples. Vitamin K, hemin and sheep blood added Brucella agar (BSBA) was used as anaerobic medium. Clinical samples were cultivated to BSBA and placed into bags with Gas-Pak (Becton Dickinson, USA) for anaerobic incubation. The BSBAs were incubated at $35 \pm 2^{\circ} \mathrm{C}$ for 48 hours; incubation time was completed to 5 days for samples without growth in 48 hours. Anaerobic and aerobic subcultures of each bacterial colony were performed. Colonies grown on BSBA were subcultured to chocolate agar and BSBA for aerotolerance testing. Chocolate agars were incubated in jars with $5 \% \mathrm{CO}_{2}$ while BSBA were incubated in anaerobic conditions. No growth in chocolate agar and growth in BSBA were evaluated as aerotolerance test positivity and the isolate was considered anaerobe. ${ }^{4}$ Antibiotic diagnostic discs - colistin $(10 \mu \mathrm{g})$, kanamycin $(1000 \mu \mathrm{g})$ and vancomycin $(5 \mu \mathrm{g})$ discs (An-Idend Discs, Oxoid, England) - were used for the first identification. The zone diameters were measured after 48 hours of anaerobic incubation. Zone diameters larger than $10 \mathrm{~mm}$ were considered sensitive (S) and those less than $10 \mathrm{~mm}$ were considered resistant (R) according to CLSI recommendations. ${ }^{4}$ Vitek-2 (Biomerioux, France) automated system was used for identification of the isolates that could not be identified with antibiotic disks. Metronidazole, imipenem, penicillin G, clindamycin and cefoxitin gradient test strips (Biomerioux, France) were used to determine the antimicrobial susceptibility of the isolates. For antibiotic susceptibility testing (AST) bacterial suspensions 
were prepared at $0.5 \mathrm{McF}$ arland turbidity (1-1.5x108), according to the manufacturer's instructions. The bacterial suspensions were homogeneously spread over BSBA and gradient test strips were placed after 15 minutes. After 48 hours of anaerobic incubation at $35 \pm 2^{\circ} \mathrm{C}$, gradient test results were interpreted according to the MIC values specified by the manufacturer. Bacteroides fragilis ATCC 25285 strain was tested for quality control during each AST.

\section{Results}

Anaerobic growth was observed in $65(8.1 \%)$ of the cultured samples, and a total of $69(8.6 \%)$ anaerobic bacteria were isolated (Table 1). Anaerobic bacterial growth was detected in $7.5 \%(47 / 620)$ of the samples sent with the syringe and $10 \%(18 / 180)$ of the samples sent via the Amies transport medium. Prevotella disiens (P.disiens) and Veillonella spp. were found to be the most frequently isolated bacteria with isolation rates of $27.5 \%$ and $20 \%$, respectively (Table 1). Anaerobic bacteria may lose their vitality when they come into contact with oxygen during subculture and antibiotic susceptibility tests. Susceptibility tests were performed in all 69 identified strains, but only 23 yielded results, while during other ASTs the bacteria could not survive. All of four Bacteroides fragilis isolates were susceptible to metronidazole, imipenem and cefoxitin; while one isolate was resistant to clindamycin and two isolates were resistant to penicillin. Of the two Clostridium spp. strains, one was resistant to clindamycin while the other was resistant to metronidazole. None of Peptostreptococcus spp. and Veilonella spp. strains showed resistance to the antibiotics tested. Clindamycin resistance was detected in 1/4 of Parvominas micra strains. While Prevotella disiensis strains had no resistance to metronidazole, imipenem, cefoxitin and clindamycin, penicillin resistance was observed in both two strains. Penicillin, clindamycin and cefoxitin resistance were observed in 1 of 3 Peptoniphilus asaccharolyticus isolates, while all 3 isolates were susceptible to imipenem and metronidazole. Fusobacterium spp. strains were susceptible to imipenem, metronidazole and cefoxitin, while both two Fusobacterium spp. isolates were resistant to penicillin.

Table I Distribution of anaerobic bacteria isolated from clinical samples

\begin{tabular}{lll}
\hline Bacteria & Number (n) & Rate (\%) \\
\hline Prevotella spp. & 19 & 27.5 \\
Veilonella spp. & 14 & 20.3 \\
Finegoldia magna & 2 & 2.9 \\
Fusobacterium spp. & 3 & 4.4 \\
Bacteroides spp. & 9 & 13.1 \\
Peptoniphilus spp. & 6 & 8.4 \\
Lactobacillus spp. & 1 & 1.4 \\
Clostridium spp. & 2 & 2.9 \\
Bifidobacterium spp. & 1 & 1.4 \\
Actinomyces spp. & 3 & 4.4 \\
Peptostreptococcus spp. & 1 & 1.4 \\
Propionibacterium spp. & 1 & 1.4 \\
Parvominas micra & 5 & 7.2 \\
Parabacteroides spp. & 2 & 2.9 \\
Total & 69 & 100 \\
\hline
\end{tabular}

\section{Discussion}

Proper sampling and transport is crucial for anaerobic cultures. ${ }^{5}$ In current study, 620 (77.5\%) samples were transported via syringes while $180(22.5 \%)$ of them were sent by Amies transport system. Anaerobic bacteria growing rate was found as $7.5 \%$ for the samples transported via syringe while it was $10 \%$ for the samples sent by Amies transport system. Transport systems such as Port-a-cul and Amies medium were shown to keep the anaerobes alive in the clinical samples for at least 24 hours. Anaerobic bacteria maintain their viability for a shorter period of time during transport of clinical samples by syringe. Transporting samples with syringes may also lead to accidental needlestick injuries. Therefore anaerobic transport systems were recommended for the transport of clinical samples. ${ }^{5}$ In our study, only anaerobic bacterial growth was observed in $6.6 \%$ of the samples, while both anaerobic and aerobic bacteria grew in $1.5 \%$ of the samples. In a study conducted by Kiremitçi et al. ${ }^{6} 5.8 \%$ of the samples grew only anaerobic bacteria while $7.8 \%$ of the samples grew both aerobic and anaerobic bacteria. ${ }^{6}$ Ercis et al. ${ }^{7}$ reported both aerobic and anaerobic growth rate of $20.5 \%$ and only anaerobic growth rate of $7.6 \% .^{7}$ In the study of Güdücüoğlu et al..$^{8}$ anaerobic bacterial growth was detected in $12.1 \%$ of the samples. ${ }^{8}$ Our study had similar growth rates with other studies when considering only the growth of anaerobic bacteria. The rates of both aerobic and anaerobic growth vary between studies.

Bacteroides, Fusobacterium, Porphyromonas and Prevotella species were reported as the most frequently isolated and the most resistant anaerobic bacteria from human samples in previous studies. ${ }^{9}$ In our study, Prevotella disiens was the most frequently isolated anaerobic bacteria with a rate of $27.5 \%$. Prevotella species $(n=49)$ were also the most frequently isolated bacteria in Boyanova et al'. studies. ${ }^{10}$ Bacteroides uniformis was reported as the most frequently isolated anaerobic bacteria in a study of Mutlu et al. ${ }^{11}$ Şengöz et al. ${ }^{12}$ reported Bacteroides species as the most common isolated anaerobic bacteria in their study. ${ }^{12}$. In the thesis study of Doğan, Bacteroides fragilis $(\mathrm{n}=6)$ was the most isolated species among 22 isolated anaerobic strains. ${ }^{13}$ Bacteroides spp. were also the most commonly isolated bacteria with a rate of $28 \%$, in a study conducted by Kiremitçi et $a 1 .{ }^{6}$ There were differences between studies when considering the frequency of isolated anaerobic bacteria. These varieties may be due to different infection sites. The bacteria isolated in our study were not compatible with other studies. In a multi-center study involving different European countries in 2019; it was stated that antibiotics usage was higher in Turkey compared to other European countries but it didn't reflect to antibiotics resistance ${ }^{14}$ This may be due to insufficient number and lack of standardization of studies on anaerobic bacteria in our country. In our study, anaerobic bacteria were mostly isolated from abscess materials. Abscesses are clinical materials in which anaerobic bacteria are most frequently isolated. Previous studies on anaerobes conducted in our country also support this data. ${ }^{4,6,12,15}$ Of the 800 specimens cultured, 228 were abscesses and anaerobic bacteria were isolated from $50(21.9 \%)$ of the abscess samples. Solely anaerobic bacteria grew in 7 (3\%) of abscess samples, while both anaerobic and facultative anaerobic bacteria grew in 43 $(18.9 \%)$ samples. In current study, Escherichia coli (E. coli) was found to be the most common facultative anaerobic agent. In Aldridge et al.'s study, similar results were obtained with our study. ${ }^{16}$ Being a member of the intestinal microbiota, E.coli facilitates the growth of anaerobic bacteria by consuming oxygen and removing it from the region. This may explain the co-existence of E. coli with anaerobic bacteria in polymicrobial infections. 
In our study, the American Food and Drug Administration (FDA) approved gradient test method was used to determine the antibiotic susceptibilities of the isolates. ${ }^{17}$ Although the gradient test method is relatively expensive compared to other AST methods, it is a practical and reliable method especially for testing a small number of isolates ${ }^{10,18}$ In a study conducted by Akhi et al. ${ }^{19}$ in 2015 , metranidazole resistance of B.fragilis group was reported as $30.8 \% .{ }^{19}$ Snydman et al. ${ }^{20}$ detected only one resistant strain to metronidazole among 5225 B.fragilis strains in the follow-up study between 1997-2004 in the USA. ${ }^{20}$ Less than $1 \%$ of the 1284 B.fragilis group (BFG) members gathered from 19 countries between 1999 and 2001 in Europe were found resistant to metronidazole..$^{21}$ In a review published in 2018, it was stated that metronidazole resistance among anaerobes increased each year and had reached dangerous levels. ${ }^{22}$ The data on metronidazole resistance in our country were quite variable. Previous studies conducted in our country reported metronidazole resistance among BFG and Peptostreptococcus as \% 0-37.5 and \% 12.5 - 23, respectively. ${ }^{4}$ Erciş et al. reported resistance to metronidazole in $14 \%$ of BFG (7). In a study conducted in a university hospital in Istanbul, metronidazole resistance among BFG, Peptostreptococcus spp., Eubacterium spp. and Mobilincus spp. were found as 4\%, 23\%, 67\% and $81 \%$, respectively. ${ }^{22}$ In current study, we found $4 \%$ metronidazole resistance in anaerobic bacteria, which is below the average of our country. The high resistance to metronidazole in domestic studies can be questioned. High metronidazole resistance may be due to metronidazole sensitivity test conditions; false resistance can be observed as a result of gradient test and media qualities and incubation conditions. ${ }^{17,23}$ On the other hand, the prevalence of nitroimidazole derivatives in patients with gastrointestinal complaints and infection prophylaxis before many surgical procedures may lead to the development of resistance to metronidazole. In a study conducted in a pediatric hospital, it was reported that metronidazole was prescribed to $17.8 \%$ of the patients of which none of parasitic agents were detected. ${ }^{24}$

Beta-lactam antibiotics have an important role in the treatment of infections due to anaerobic bacteria. However, especially Bacteroides species are highly resistant to beta-lactams. ${ }^{22}$ In a study conducted by Jeverica et al. ${ }^{25}$ BFG isolates were reported to be resistant to penicillin, amoxicillin clavulanate and imipenem with rates of $99 \%, 8 \%$ and $1 \%$, respectively. ${ }^{25}$ In our study, penicillin resistance was observed in 7/23 of all anaerobic isolates. Lewis et al. reported penicillin resistance among 5 isolates of P.melaninogenica, and 26 of 39 Prevotella species. ${ }^{26}$ Cefoxitin, cefotetan and cephamycin are cephalosporin group antibiotics that are more effective on anaerobic bacteria than penicillin. Resistance to these cephalosporin antibiotics has also developed in the last decades. ${ }^{3,24}$ Akhi et al. ${ }^{19}$ reported $38.5 \%$ resistance rate to cefoxitin among BFG. ${ }^{19}$ Previous studies in Turkey reported cefoxitin resistance among BFG in a range from $12 \%$ to $81.8 \%{ }^{7,8,10}$ In current study, cefoxitin resistance was not observed in B. fragilis isolates. Clindamycin resistance tends to increase among anaerobes in the last decades. In a study about antibiotic resistance among BFG, Akhi et al. ${ }^{19}$ reported clindamycin resistance as $38.5 \% .{ }^{19}$ Jeverica et al. reported clindamycin resistance of $27 \%$ among BF. ${ }^{25}$ In the study conducted by Kiremitçi et al. ${ }^{6}$ a high rate of $(8 / 15)$ clindamycin resistance was reported in Bacteroides isolates. ${ }^{6}$ In current study, clindamycin resistance was found in 4 of 23 anaerobes and in one isolate of 4 Bacteroides spp. strain. Empirical treatment of upper respiratory tract infections with clindamycin may have contributed to this resistance. ${ }^{29}$ In our study, imipenem resistance was not detected. This data was found to be compatible with studies in our country and worldwide. ${ }^{28,30}$ Infections related to anaerobes are important factors that prolong hospital stay after surgery, especially in surgical clinics. Increased resistance to anaerobic infections has been observed in recent decades. Anaerobic bacteria should be identified and regional antibiotic resistance should be determined in order to prevent antibiotic resistance. Although cultivation and performing resistance tests of anaerobes seem troublesome, it is crucial to identify and perform AST for anaerobes.

\section{Acknowledgments}

None.

\section{Conflicts of interest}

The author declare that there is no conflict of interest.

\section{References}

1. Gürler N. Anaerobic infections and laboratory diagnosis. Ankara. 2005:934.

2. Gürler N. Overview of anaerobic infections and antimicrobial resistance ANKEM. 2001;15(3):593-599.

3. Schuetz AN. Anaerobic Bacteria: Antimicrobial Susceptibility Testing and Resistance Patterns. Antimicrobial Resistance in the 21st Century. Springer; 2018:191-215.

4. Türkkan AA. Identification anaerobic bacteria isolated from clinical samples and determination of antibiotic suspectibilities by E-test methods. Eskişehir Osmangazi University; 2008.

5. Citron DM, Warren YA, Hudspeth MK, et al. Survival of aerobic and anaerobic bacteria in purulent clinical specimens maintained in the Copan Venturi Transystem and Becton Dickinson Port-a-Cul transport systems. $J$ Clin Microbiol. 2000;38(2):892-894.

6. Kiremitçi A, Türkkan A, Akgün Y, et al. Isolation and Antimicrobial Susceptibility of Anaerobic Bacteria Isolated from Clinical Samples. ANKEM. 2008;22(3):132-144.

7. Ercis S, Tunçkanat F, Hasçelik G. Anaerobic Bacteria Isolated From Patients With Suspected Anaerobic Infections. Mikrobiol Bul. 2005;39:447-454.

8. Güdücüoğlu H, Bozkurt H, Bayram Y, et al. Microorganisms isolated from bile samples of the patients who had cholesistectomy and their antibiotics susceptibilities. Genel Tlp Derg. 2004;14(1):13-17.

9. Bozkurt H, Güdücüoğlu H, Bayram Y, et al. The Anaerobic bacteria isolated from various clinical specimens and their antibiotic susceptibilities. Van Tip Derg. 2004;11:85-91.

10. Mutlu E, Yücesoy M. Determination of antibiotic susceptibilities and B-Lactamase activity of anaerobic bacteria by agar dilution and E-test methods. Infeksiyon Derg. 2003;17:275-280.

11. Boyanova L, Kolarov R, Gergova G, et al. Anaerobic bacteria in 118 patients with deep-space head and neck infections from the University Hospital of Maxillofacial Surgery, Sofia, Bulgaria. J Med Microbiol. 2006;55(9):1285-1289.

12. Şengöz G, Yaşar K, Berzeg D, et al. Anaerobic Bacteria Isolated from Clinical Samples and Their Antibiotic Susceptibilities. Türk Mikrobiyol Cem Derg. 2005;35:107-113.

13. Dogan M. The identification of anaerobic bacteria isolated from various clinical materials and determination of antıbıtıc susceptibility. Selcuk University Faculty of Medicine; 2008.

14. Veloo A, Tokman HB, Jean-Pierre H, et al. Antimicrobial usceptibility profiles of anaerobic bacteria, isolated from human clinical specimens, within different European and surrounding countries. A joint ESGAI study. Anaerobe. 2019;61:102111 
15. Toprak NU, Veloo AC, Urban E, et al. A multicenter survey of antimicrobial susceptibility of Prevotella species as determined by Etest methodology. Anaerobe. 2018;52:9-15.

16. Aldridge K. Anaerobes in polymicrobial surgical infections: incidence, pathogenicity, and antimicrobial resistance. European J Surgery Supplement. 1994;573:31-37.

17. Brook I, Wexler HM, Goldstein. Antianaerobic antimicrobials: spectrum and susceptibility testing. Clin Microbiol Rev. 2013;26(3):526-546.

18. Demir C. Typification and Determination of Antibiotic Ausceptibility Profiles with E Test Methods of Anaerobic Gram Negative Bacilli Isolated from Various Clinical Specimen. Afyon Kocatepe University, Institute of Health Sciences; 2017.

19. Akhi MT, Ghotaslou R, Beheshtirouy S, et al. Antibiotic susceptibility pattern of aerobic and anaerobic bacteria isolated from surgical site infection of hospitalized patients. Jundishapur J Microbiol. 2015;8(7):e20309.

20. Snydman D, Jacobus N, McDermott L, et al. National survey on the susceptibility of Bacteroides fragilis group: report and analysis of trends in the United States from 1997 to 2004. Antimicrob Agents Chemother. 2007;51(5):1649-1655.

21. Hedberg M, Nord CE. Antimicrobial susceptibility of Bacteroides fragilis group isolates in Europe. Clin Microbiol Infection. 2003;9(6):475-488.

22. Toprak NÜ. Susceptiblity Studies. In: XXXII Turkish Microbiology Congress 2006: 335-339.

23. Alauzet C, Lozniewski A, Marchandin HJA. Metronidazole resistance and nim genes in anaerobes: A review. Anaerobe. 2019;55:40-53.
24. Bacalan F. Çakır F, Demirkaya S, Özcan N. Viral and Parasitic Gastroenteritis Agents and Metronidazole Treatment in Diyarbakir Children's Hospital. FLORA J Infect and Clin Microbiol. 2019;24(3):227235 .

25. Jeverica S, Kolenc U, Mueller-Premru M, et al. Evaluation of the routine antimicrobial susceptibility testing results of clinically significant anaerobic bacteria in a Slovenian tertiary-care hospital in 2015. Anaerobe. 2017;47:64-69.

26. Lewis M, Parkhurst C, Douglas C, et al. Prevalence of penicillin resistant bacteria in acute suppurative oral infection. J Antimicrob Chemother. 1995;35(6):785-791.

27. Botta GA, Arzese A, Minisini R, et al. Role of structural and extracellular virulence factors in gram-negative anaerobic bacteria. Clin Infect Dis. 1994;18(Supp 4):260-264.

28. Demir C, Keşli R. Identification of Anaerobic Gram-Negative Bacilli Isolated from Various Clinical Specimens and Determination of Antibiotic Resistance Profiles with E-Test Methods. Microbiol Bul. 2018;52(1):7279.

29. Hacımustafaoğlu MK, Gürler N, Ünal. Antimicrobial Resistance in the Planning of the Empirical Therapy of Upper Respiratory Tract Infections: Current Situation and Treatment Strategies in Turkey and in the World. ANKEM. 2018;32(3):109-130

30. Tan TY, Ng LSY, Kwang LL, et al. Clinical characteristics and antimicrobial susceptibilities of anaerobic bacteremia in an acute care hospital. Anaerobe. 2017;43:69-74. 